\title{
Biopsy utility in the workup of ANCA-associated vasculitis
}

\author{
Carlos Anjo, Hernando Campos, Sofia Narciso, Glória Nunes da Silva, Fátima Duarte \\ Hospital Pulido Valente, Centro Hospitalar Lisboa Norte, Lisbon, Portugal
}

Received: October 17, 2018

Accepted: November 27, 2018 Online Published: December 5, 2018

DOI: $10.5430 /$ crim.v6n1p5

URL: https://doi.org/10.5430/crim.v6n1p5

\begin{abstract}
ANCA - associated Vasculitis (AAV) positive biopsy is supportive of vasculitis diagnosis, the aim of this manuscript is that renal biopsy is not always compulsory in the diagnosis and management of AAV. 79 years old, Caucasian women, admitted with AAV suspicion, MPO positive. A Microscopic polyangiitis with a pulmonary - renal syndrome was diagnosed after other AAV were excluded. Remission induction and maintenance therapy was made. In 6 months the patient presented a considerable improvement with lower MPO value, ESR and a stabilization of kidney function. Renal biopsy has to be performed in the majority of patients with the suspicion or renal AAV, although in this case it was not performed considering that the patient was on chronic anticoagulation and renal biopsy was not essential for the diagnosis. We suggest that renal biopsy is a valuable method in establishes the aetiology of kidney disease and that it could be dismissed in AAV with suggestive clinical presentation and low suspicion for secondary vasculitis.
\end{abstract}

Key Words: Renal biopsy, Microscopic Polyangiitis, Pulmonary - renal syndrome, ANCA - associated vasculitis, Autoimmune disease

\section{INTRODUCTION}

Anti-neutrophil cytoplasmic antibody (ANCA)-associated vasculitis (AAV) is the most frequent aetiology of rapidly progressive glomerulonephritis syndrome ${ }^{[1]}$ and the incidence of vasculitis-related mortality is actually 0.10 per 100 persons. ${ }^{[2]}$ Soon after the outspread of ANCA testing a discussion began as to whether biopsy can be avoided in AAV, however, renal biopsy remains the definitive complementary exam and should always be considered in the AAV diagnostic workup. ${ }^{[1]}$ Although percutaneous kidney biopsy (PKB) is a safe procedure, complications can occur and are mainly related to bleeding. ${ }^{[3]}$ The aim of this manuscript is that renal biopsy is not always compulsory in the diagnosis and management of AAV.

\section{CASE REPORT}

We present 79 years old women, dependent in activities of daily living, currently living in a care home. With known history of Sarcoidosis, Sjögren Syndrome, Atrial Fibrillation, Parkinson Disease, Hypotiroidism, and left hemiparesis Stroke related. The patient was medicated with warfarin, levodopa/carbidopa 200/50 mg, ropinirol $4 \mathrm{mg}$, levotiroxyn $50 \mathrm{mcg}$ and pantoprazole $20 \mathrm{mg}$. It was admitted with easy fatigability, no visible haemorrhage, fever, arthralgia, myalgia or Raynaud phenomenon. At the physical examination, the patient was hemodynamically stable (arterial blood pressure: $125 / 75 \mathrm{mmHg}$, heart rate: $75 \mathrm{mmHg}, \mathrm{SaO}_{2} 97 \%$ with $\mathrm{fiO}_{2} 21 \%$, axillary temperature $36.5^{\circ} \mathrm{C}$ ), oriented, calm, no oral ulcerations, lymphadenopathy or skin lesions. Auscul-

\footnotetext{
*Correspondence: Carlos Anjo; Email: carloslccanjo@gmail.com; Address: Medicina III Hospital Pulido Valente, Alameda das Linhas de Torres, 117, 1769-001, Lisbon, Portugal.
} 
tation of the lungs was clear bilaterally, and the heart had a normal rhythm, no carotid or abdominal bruits were heard. Carotid, radial, posterior tibialis and pedal pulses were symmetric and no peripheral oedema was observed. In blood sample at admission (see Table 1), we observed a microcytic hypochromic anaemia, normal ferritin levels, increased erythrocyte sedimentation rate and PCR, decreased renal function, INR in therapeutic range and normal platelets count, in the urine sample it was observed important haematuria and proteinuria.

Table 1. Patient's blood and urine sample at admission and after treatment

\begin{tabular}{lcc}
\hline & Admission & After Treatment \\
\hline Haemoglobin (g/dl) & 7.7 & 11.1 \\
VGM (fl) & 77.7 & 91.2 \\
ESR (mm) & 91 & 53 \\
PCR (mg/l) & 4.5 & 0.7 \\
Ferritin (ug/dl) & 84.9 & 79.1 \\
Creatinin (mg/dl) / & & \\
Creatinin Clearance & $1.4 / 29.3$ & $1.1 / 41$ \\
(ml/min) & & \\
INR & 2.4 & 2.6 \\
Haematuria (/ul) & $>1,800$ & 158.4 \\
24h Proteinuria & $1,271.4$ & 524 \\
(mg/dl) & & \\
\hline
\end{tabular}

Table 2. Patient's autoimmunity and viral serology

\begin{tabular}{lcc}
\hline & Admission & After Treatment \\
\hline ANA & $\begin{array}{c}1 / 1280 \\
\text { (speckled } \\
\text { pattern) }\end{array}$ & $\begin{array}{c}1 / 160 \text { (speckled } \\
\text { pattern) }\end{array}$ \\
MPO ANCA (UQ) & 196.6 & 51.1 \\
PR3 ANCA & Negative & Negative \\
dsDNA & Negative & Negative \\
GBM antibody & Negative & Negative \\
Cryoglobulinaemia & Negative & Negative \\
HIV & Negative & Negative \\
AgHBs & Negative & Negative \\
HCV antibody & Negative & Negative \\
\hline
\end{tabular}

Based on a vasculitis suspicion, specific laboratory exams were performed (see Table 2), ANA 1/1,280 (with speckled pattern), MPO 196.8UQ, negative PR3, dsDNA, GBM antibody, cryoglobulinaemia, HIV, AgHBs HCV antibody, VDRL and 1,271.4 mg/dl proteinuria in $24 \mathrm{~h}$ urinalysis. Chest radiograph presented a normal pattern (see Figure 1), thoracoabdominal CT scan with small pleural effusion and ground glass appearance (see Figure 2). A bronchoalveolar lavage was performed, abundant hemosiderophages were observed and lung biopsy was inconclusive. Renal echographic study with preserved kidneys size, with a slight decrease in renal parenchymal thickness. Renal biopsy was not performed based in procedure risk/benefits. A microscopic polyangiitis with a pulmonary - renal syndrome was assumed and treatment was started, $1 \mathrm{~g}$ /day metilprednisolone bolus for 3 days, 6 cyclophosphamide pulses and 7 plasma exchange cycles were performed as remission induction treatment. Prednisone starting with $1 \mathrm{mg} / \mathrm{kg} /$ day and azathioprine $2 \mathrm{mg} / \mathrm{kg} /$ day were performed as maintenance treatment.

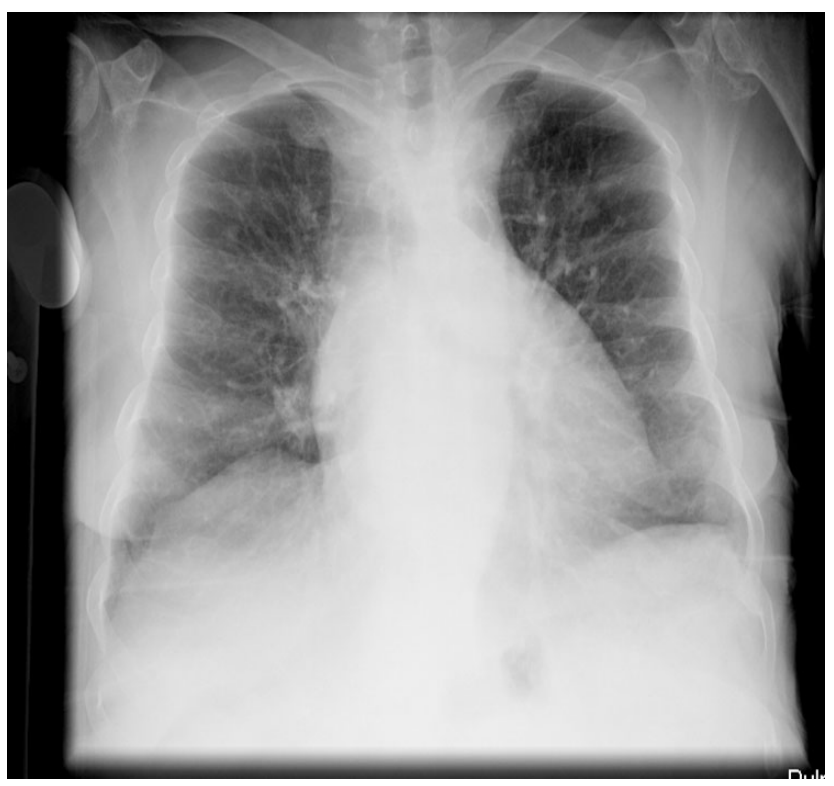

Figure 1. Chest Radiography with normal pattern

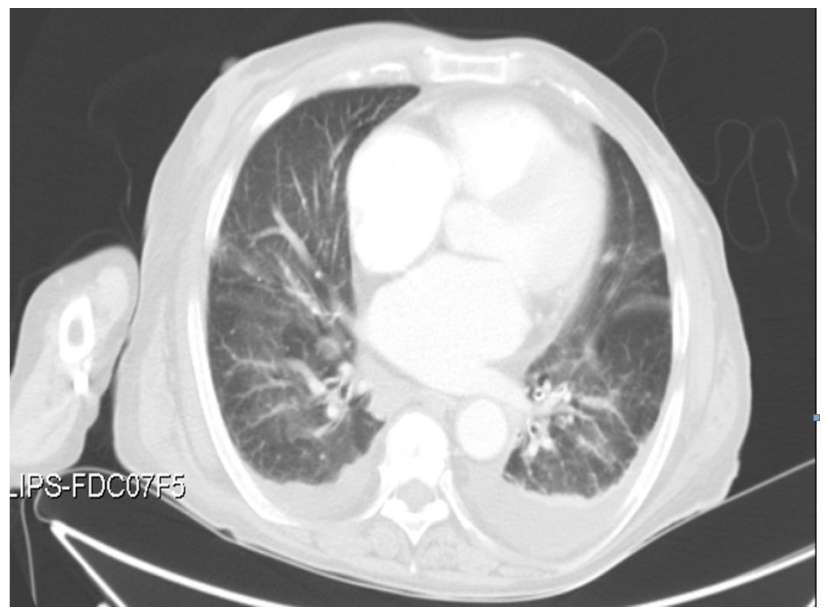

Figure 2. Thoracoabdominal CT scan with small pleural effusion and ground glass appearance

During the follow up period the patient remains stable and it was observed haemoglobin, $24 \mathrm{~h}$ haematuria, proteinuria and renal function improvement (see Table 1). ANCA MPO presented also lower levels (see Table 2). Control chest radiography also presented a normal pattern in the follow up period. 


\section{DISCUSSION}

We present 79 years old women with an ANCA vasculitis, specifically a microscopic polyangiitis with a pulmonary renal syndrome. No renal biopsy was performed because it was an elderly patient on anticoagulation and it was no doubt about the diagnosis. No eosinophilia, pulmonary nodules or infiltrates were observed, therefore, eosinophilic granulomatosis with polyangiitis was excluded. MPO ANCA vasculitis is presented in $60 \%$ to $85 \%$ of microscopic polyangiitis patients and less then $10 \%$ in granulomatosis with polyangiitis, ${ }^{[4]}$ also no pulmonary nodules or infiltrates were observed, granulomatosis with polyangiitis was excluded and microscopic polyangiitis was proposed as the most probable diagnosis. The 2016 EULAR (European League Against Rheumatism) recommendations for the management of AAV declares that a positive biopsy is strongly supportive of vasculitis and biopsies are recommended to assist in establishing a new diagnosis, this recommendation has a low level of evidence and a low grade of recommendation. ${ }^{[5]}$ Renal biopsy can be omitted when there is a common presentation of renal vasculitis with positive PR3 or MPO ANCA antibody and when there is a low suspicion for a secondary vasculi- tis, false positive, or vasculitis mimic. ${ }^{[1]}$ Based on literature we can afirm that renal biopsy could be dismissed, athough this approach can be true, overall, renal biopsy has to be performed in the majority of patients with the suspicion or renal AAV. Percutaneous kidney biopsy is a valuable method to establish the aetiology of kidney disease and it could be indicated when MPO - ANCA assays are borderline or in further evaluation of relapsing vasculitis. ${ }^{[1]}$

Complications in kidney biopsy can range in severity from minimal to catastrophic like nephrectomy or even death, ${ }^{[3]}$ to assess renal biopsy risks/benefits in patients on chronic anticoagulation it should be considered whether it is essential for diagnosis, prognosis and management, in this case it was not. $^{[6]}$

\section{ACKNOWLEDGements}

To the Department of Autoimmune Diseases from the Hospital Clinic de Barcelona (Catalonia, Spain), Dr. José Rodríguez-Hernández, Dr. Sergio Prieto-González, Dr. Gerard Espinosa and Dr. Ricard Cervera.

\section{CONFLicts OF INTEREST Disclosure}

The authors have declared no conflicts of interest.

\section{REFERENCES}

[1] Jayne D. Vasculitis - when can biopsy be avoided? Nephrol Dial Transplant. 2017; 32(9): 1454-1456. https://doi.org/10.109 3/ndt/gf 248

[2] Jardel S, Puéchal X, Le Quellec A, et al. Mortality in systemic necrotizing vasculitides: A retrospective analysis of the French Vasculitis study group registry. Autoimmun Rev. 2018; 17(7): 653-659. https://doi.org/10.1016/j . autrev. 2018.01.022

[3] Whittier WL. Complications of the percutaneous kidney biopsy. Adv Chronic Kidney Dis. 2012; 19(3): 179-187. https ://doi.org/10 $.1053 /$ j. ackd.2012.04.003
[4] Cervera R. Enfermedades Autoinmunes sistémicas diagnóstico y tratamiento (5th ed.). Madrid: Editora Medica Panamericana. 2015.

[5] Yates M, Guillevin L, Cid MC, et al. EULAR/ERA-EDTA recommendations for the management of ANCA-associated vasculitis. Ann Rheum Dis. 2016; 75(9): 1583-1594. https://doi.org/10.113 $6 / \operatorname{ard} .2008 .088096$

[6] Shidham GB, Siddiqi N, Beres JA, et al. Clinical risk factors associated with bleeding after native kidney biopsy. Nephrology (Carlton). 2005; 10(3): 305-310. https://doi.org/10.1111/j.1440-179 7.2005.00394.x 